\title{
CROSS-REFERENCE MAXIMUM LIKELIHOOD ESTIMATE RECONSTRUCTION FOR POSITRON EMISSION TOMOGRAPHY
}

\author{
Chung-Ming Chen, Henry Horng-Shing Lu*, And Yun-PAi Hsu* \\ Institute of Biomedical Engineering, National Taiwan University, Taipei, \\ *Institute of Statistics, National Chiao Tung University, Hsinchu, Taiwan, R.O.C.
}

\begin{abstract}
Maximum likelihood estimate (MLE) is a widely used approach for PET image reconstruction. However, it has been shown that reconstructing emission tomography based on MLE without regularization would result in noise and edge artifacts. In the attempt to regularize the maximum likelihood estimate, we propose a new and efficient method in this paper to incorporate the correlated but possibly incomplete structure information which may be derived from expertise, PET systems or other imaging modalities. A mean estimate smoothing the MLE locally within each region of interest is derived according to the boundaries provided by the structure information. Since the boundaries may not be correct, a penalized MLE using the mean estimate is sought. The resulting reconstruction is called a cross-reference maximum likelihood estimate (CRMLE). The CRMLE can be obtained through a modified EM algorithm, which is computation and storage efficient. By borrowing the strength from the correct portion of boundary information, the CRMLE is able to extract the useful information to improve reconstruction for different kinds of incomplete and incorrect boundaries in Monte Carlo studies. The proposed CRMLE algorithm not only reduces the estimation errors, but also preserves the correct boundaries. The penalty parameters can be selected through human interactions or automatically data-driven methods, such as the generalized cross validation method.
\end{abstract}

Biomed Eng Appl Basis Comm, 2001 (August); 13: 190-198.

Key Words: Maximum likelihood estimate, generalized EM algorithm, regularization, generalized cross-validation.

\section{INTRODUCTION}

Positron Emission Tomography (PET) is a functional imaging modality providing biochemical, physiological, and metabolic information in the human body. Like X-ray CT, the distribution of radioisotopelabeled chemicals may be estimated from the measured gamma photon pairs through filtered-backprojection algorithms as adopted by most commercial PET systems accredited to their computational efficiency.

Received: July 7, 2001; accepted: July 30, 2001 Correspondence: Chung-Ming Chen, Institute of Biomedical Engineering, National Taiwan University. Taipei, Taiwan, R.O.C.
However, since the filtered-backprojection algorithms were originally designed for X-ray CT, many assumptions made for these algorithms do not hold for PET image reconstruction. As a result, the reconstructed images, especially those with small features, may not be accurate enough for clinical use [1].

To overcome the potential problems inherent in the filtered-backprojection algorithm, various approaches based on maximum likelihood with EM algorithms have been proposed for PET image reconstruction, e.g., Shepp and Vardi [2], Lange and Carson [3], Vardi, Shepp and Kaufman [4], Politte and Snyder [5], Fessler et al. [6]. Theoretically, the physical process of a PET system may be modeled as a Poisson random process that suggests the random observations in a PET as Poisson random variables. Morcover, the mean of these random variables is indirectly related to 
the target image intensity by a linear transformation. Under this model, there are at least two sources of errors intrinsic in the reconstruction of PET images. One is due to the random variation of Poisson random variables. This can be handled via the maximum likelihood approach as attempted by [2-6]. A good review on the MLE approaches may be found in the book by Snyder and Miller [7].

The other source of error is caused by the illposedness in inverting the linear transformation. This can be managed by the regularization methods [8-10]. Snyder et al. [11] demonstrated that the MLE without regularization will show the noise and edge artifacts. Therefore, it is important to regularize MLE for a better reconstruction. A variety of regularization methods have been studied in literature, like the early stopping rule in Veklerov and Llacer [12], the method of sieves in Snyder and Miller [13], the Bayesian approaches with different kinds of priors in Hebert and Leahy [14], Green [15], Herman et al. [16], and so on. Ouyang et al. [17] proposed to use the correlated structure information as the prior information and obtain the Bayesian reconstruction of PET via the "weighted line site" method in their series of studies. While the correlated structure information contains more information about boundarics than the mathematical form of regularization does, the Bayesian reconstruction needs more computational efforts than the MLE-EM approaches. Therefore, in this paper, we would like to investigate a new and efficient approach to integrate the correlated but incomplete structure information using the MLEEM approach. The corrclated boundary information is obtained from an expert, an informed audience or the same or other tomography systems such as transmission PET, X-ray $C T$ scan, and MRI. The intensities within the boundaries arc not necessarily uniformly distributed. The proposed method, which is named as a cross-reference maximum likelihood estimate (CRMLE), has been shown to not only fully utilize the correlated structure information to obtain a better reconstruction, but also retain the computational efficiency of the MLE-EM approach in contrast to the Bayesian approach.

This paper is organized as follows. The model of PET, the ill-posedness, stability, and finite sample behaviors of MLE will be investigated in Section 2. Because of these phenomena, we will also discuss some versions of regularization based on the MLE in Section 2. Via incorporating the information of local smoothness, we can get better reconstruction images after cross-referring the correlated information. This new method, CRMLE, will be introduced in Section 3. The implementation issues such as the influences and selection rules of penalty parameters will be investigated in Section 4. The implementation results and discussions are also presented in this section. Conclusions and future studies are provided in Section 5.

\section{PET MODEL AND MLE BEHAVIOR}

Consider the phantom illustrated Figure 1. Suppose that there are three anatomic boundaries (ellipses) that define three regions, each of which may be an organ or a portion of a tissue. The emission intensity in one region may be homogencous within its boundary as in the upper and right regions of Figure 1. That is, the functional boundaries of emission intensities are the same as the anatomic boundaries in the upper and right regions. But, if some part of a region becomes abnormal, the emission intensity will be inhomogeneous within the region as the left region of Figure 1. The functional boundaries are thus different from the anatomic boundaries. It is aimed to use the information of anatomic boundaries to enhance the reconstructed images without destroying the functional boundaries.

The model of the PET system employed in this studied follows that used in [2]. Let $\lambda$ denote the image to be reconstructed, which is decomposed into $B$ square boxes (or pixels). The number of emission photon pairs generated in box $b, n(b)$, is assumed to be Poisson distributed with a mean, $\lambda(b)$, for $b=1$, $2, \ldots$, B. Owing to the random variation of Poisson random variables, the actual number of emission, $n(b)$, may be quite different from the mean, $\lambda(b)$. In particular, for a large value of $\lambda(b)$, the variation is large since the variance of a Poisson random variable is equal to the mean.

When a positron radiates and annihilates with a nearby electron, two photons are generated and traveled in almost an opposite direction along a line. If two detectors within a preset time window receive these two photons, an ..annihilation event is identified in the tube formed by these two detectors. Let $D$ denote the total number of tubes. For a PET system with $N_{d}$ distinct detectors, there can be at most $\left(\begin{array}{c}N_{d} \\ 2\end{array}\right)$ tubes.

Unlike the filtered-backprojection algorithms that assume a space-invariant point spread function in generation of projection data, the "space-variant notion" is incorporated in the PET model employed in this study

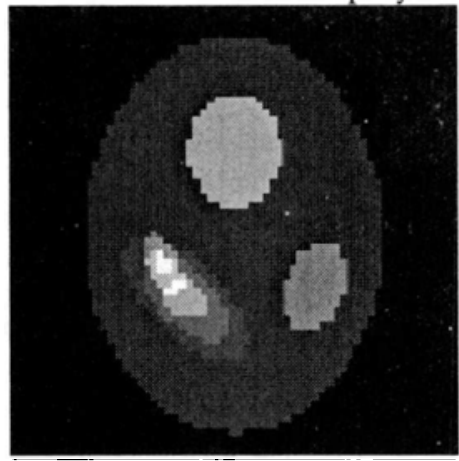

Fig. 1 Test phantom 1 is shown. 
that may describe the physical process more closely. Each box $b$ is associated with a value, $p(b, d)$, as the probability for a pair of photons generated in the box $b$ and detected by the tube $d$, where $b=1,2, \ldots, B$ and $d=1,2, \ldots, D$. One possible assignment for $p(b, d)$ would be the angle of view from box $b$ to tube $d$. Without loss of generality,

$$
p(b, \cdot)=\sum_{d=1}^{D} p(b, d) \text { is assumed to be } 1 .
$$

According to the "thinning" property of Poisson random variables $[4,18]$, the total number of coincidences detected by the $d$-th tube, $n(d)$ is an independent Poisson random variable,

$$
n^{*}(d) \sim \operatorname{Poisson}\left(\lambda^{*}(d)\right)
$$

where ' ' means 'distributed as,' and

$$
\lambda^{*}(d)=\sum_{b=1}^{B} \lambda(b) p(b, d)
$$

To see the performance of MLE in finite samples, Monte Carlo studies are simulated. Suppose the target phantom is as Figure 1 . The number of boxes, $B$, is equal to $N_{b}{ }^{*} N_{b}=64^{*} 64$ and the number of detectors is $N_{d}=64$. The MLE can be reconstructed by the EM algorithm in Shepp and Vardi [2]. The log-likelihood of MLE-EM with respect to the iteration number is plotted in Figure 2. The log-likelihood is non-decreasing as the iteration number increases, which is assured by the property of EM algorithm. However, the loglikelihood of the true phantom is not the same as the converging value via the MLE-EM. At some iteration number, like 19 in this case study, the log-likelihood of MLE-EM would be closest to that of the true phantom. But as the iteration number increases further, the loglikelihood will be more and more away from that of the true phantom! To obtain the best reconstructed image before it deteriorates, Veklerov and Llacer [12] discussed the stopping rule based on statistical hypothesis testing. However, it is difficult to choose a proper stopping time in practice. Even if we select a good stopping time, such as 19 iterations in Figure 2, the reconstructed image in Figure 3 is still blurred without sharp boundaries and fine local structures. As the number of iterations increases, the reconstructed image becomes more and more blurring and spiky!

This is a commonplace phenomenon for MLE approaches. Snyder et al. [11] showed that edge and noise artifacts would be incurred due to the illposedness of PET. A good alternative to reduce the edge and noise artifacts is to use the regularization methods. Several regularization methods have been proposed previously. Silverman et al. [19] and Green [20] added in a smoothness penalty in the regularization method and modify the EM algorithm accordingly. Snyder and Miller [13] used the method of sieves together with the EM algorithm. Green [15] also consid- ered the modified EM algorithm for Bayesian approach with prior information about the patterns of target images. In addition, the minimax estimator based on tapered orthogonal series in Johnstone and Silverman [21], the adaptive constrained methods of regularization estimator in Lu and Wells [10] are also proposed among many others.

To see the effects of regularization, a penalized MLE (PMLE) with a global penalty is demonstrated. Instead of maximizing the log-likelihood, one minimizes the negative value of $\mathrm{log}$-likelihood and a 2-norm penalty. That is, this PMLE is

$$
\dot{\lambda}_{\text {PMLE }}=\arg \min _{\lambda=0}\left\{-l(\lambda)+\alpha\|\lambda\|^{2}\right\} \text {, }
$$

where $\mathrm{l}(\lambda)$ is the log-likelihood, $\alpha$ is a positive penalty parameter and $\|\lambda\|$ is the 2-norm of $\lambda$. If $\alpha \rightarrow 0$, then $\hat{\lambda}_{\text {CRMLE }} \rightarrow \hat{\lambda}_{\text {MLE }}$ On the other hand, if $\alpha \rightarrow \infty$, then

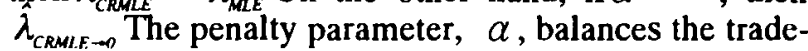
off between the log-likelihood and the penalty term.

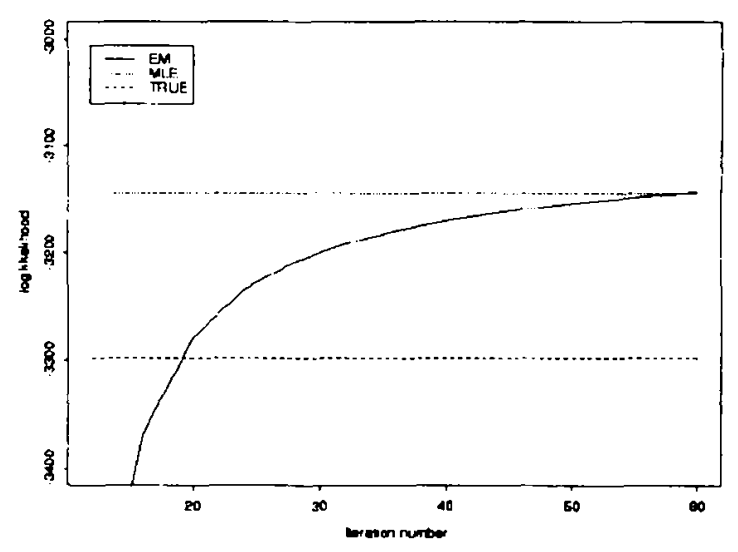

Fig. 2 The log-likelihoods of the MLE-EM, the converging MLE, and the true phantom with respect to the iteration numbers are displayed.

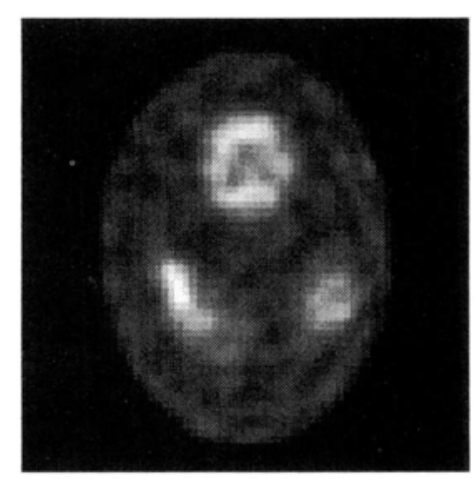

Fig. 3 The MLE-EM reconstruction is displayed with 19 iterations. 
The EM algorithm can be applied to find the PMLE. The updating formula becomes

$$
\lambda^{n+w}(b)=\frac{-1+\sqrt{1+8 \alpha \hat{n}(b, .)}}{4 \alpha},
$$

where

$$
n(b, .)=\lambda^{\text {old }}(b) \sum_{-=1}^{D} \frac{p(b, d) n^{*}(d)}{\sum_{b^{\prime}=1}^{R} p\left(b^{\prime}, d\right) \lambda^{\text {old }}\left(b^{\prime}\right)}
$$

Note that this is the unique nonnegative solution in the $M$ step. The resulting estimates will be different for a different $\alpha$. The log-likelihoods and 2-norm differences of MLE, PMLE and the true phantom can be plotted. If we choose $\alpha$ equal to the minimum value, 0.0001 in this case, then the PMLE has a less 2-norm difference with respect to the true phantom than that of the MLE. But the improvement is not too much as one can see that the PMLE in Figure 4 is about the same as the MLE in Figure 2. If the penalty $\alpha$ is chosen too large, then the PMLE will approach 0 and have even

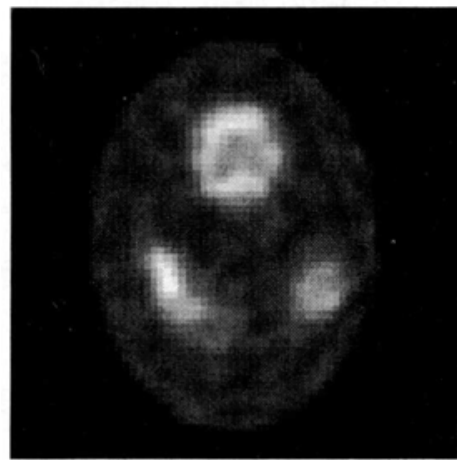

Fig. 4 The PMLE-EM reconstruction is displayed with penalty parameter 0.0001 and 19 iterations.

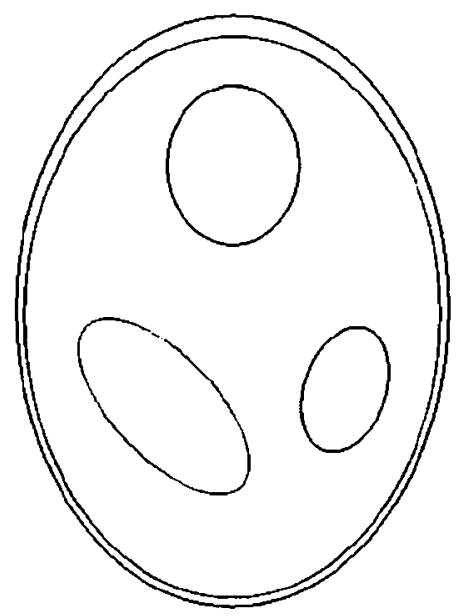

Fig. 5 The incomplete boundary information is shown. bigger 2-norm differences with respect to the true phantom than those of the MLE. Therefore, the selection of the penalty parameter is important.

The choice of penalty term is substantial as well. Green [20] suggested another form of penalty form, $\sum \log \left\{\cosh \left(\lambda\left(b_{0}\right)-\lambda\left(b_{1}\right)\right\}\right.$, where the sum is taken over the neighboring pixel pairs. The resulting EM algorithm is difficult to obtain a closed form solution in the M-step. He proposed the One-Step-Late (OSL) algorithm that approximates the current solution via the previous solution in the M-step. These penalized terms can also be interpreted in the Bayesian framework. Geman and Geman [22] used the global energy function with Gibbs sampling technique to obtain the Bayesian reconstruction. Ouyang et al. [17] tried to incorporate the local smoothness contained in the boundaries to the Bayesian reconstruction by Gibbs samplings. In order to achieve this goal, they need to consider the encrgy function in the presence of line site induced by boundaries. While it is appealing in combining the local smoothness information, the computation and complexity is quite demanding. In this paper, we propose a more efficient way to make use of the local structure as presented in the following section.

\section{CROSS-REFERENCE MLE-EM}

Since a boundary defines a region that is likely to have homogeneous emission intensities, we can get a mean estimate, $\hat{\lambda}_{\text {MFAN }}$, by doing the local average of the MLE within the boundaries. For instance, if the boundaries are as in Figure 5 , the mean estimate is as Figure 6 . However, the anatomical boundary may not be consistent with the functional boundary. That is, the boundary information may be incomplete. For example, the boundaries in Figure 5 do not contain all the boundaries in Figure 1. Thus, the mean estimate is only a rough estimate for the purpose of reference. In order to retain the local structure in the MLE, one needs to cross-refer the MLE and the mean estimate. Thus, we define the Cross-Reference MLE (CRMLE) as

$$
\lambda_{\text {CRMLE }}=\arg \min _{\lambda \geq 0} \Phi(\lambda),
$$

where

$$
\Phi(\lambda)=-l(\lambda)+\alpha\left\|\lambda-\dot{\lambda}_{\text {MEAN }}\right\|^{2},
$$

$\alpha>0$ and 2-norm is used for the penalty term. Suppose $\alpha \rightarrow 0$, then $\hat{\lambda}_{\text {CRMLE. }} \rightarrow \hat{\lambda}_{\text {MI.E. }}$.If $\alpha \rightarrow \infty$, then $\hat{\lambda}_{\text {CRMLE }} \rightarrow \hat{\lambda}_{\text {MEAE }}$. The resulting images by the CRMLE for one penalty parameter are shown in Figure 7 . It is evident from Figure 7 that the CRMLE can borrow the strength from the local smoothness information via choosing a proper penalty parameter.

The Lagrangian function can be obtained after introducing the Lagrangian multiplier, $\beta=(\beta(1), \beta(2)$, 


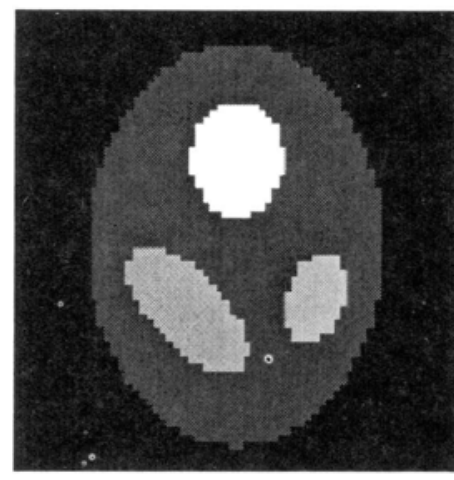

Fig. 6 The mean estimate based on the boundary information and MLE-EM.

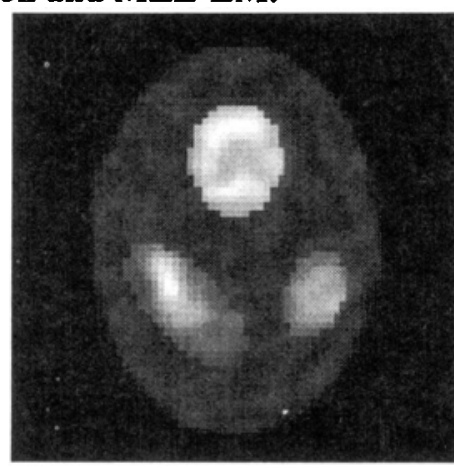

Fig. 7 The CRMLE-EM reconstruction is displayed with penalty parameter 0.0002 and 15 iterations.

$\ldots, \beta(B))^{T}$

$\Psi=-l(\lambda)+\alpha\left\|\lambda-\dot{\lambda}_{\text {MFAN }}\right\|^{2}-\beta^{T} \lambda$.

If the MLE is required to be nonnegative, then the Kuhn-Tucker (KT) conditions can be applied to find the MLE [18]. The KT conditions are listed as follows.

1. $\lambda(b) \geq 0$ for all $b$,

2. $\left.\frac{\partial l(\lambda)}{\partial \lambda(b)}\right|_{i} \leq 2 \alpha\left(\hat{\lambda}(b)-\hat{\lambda}_{\text {MEAN }}(b)\right)$,

3. $\left.\lambda(b) \frac{\partial l(\lambda)}{\partial \lambda(b)}\right|_{\dot{\lambda}}=2 \alpha\left(\hat{\lambda}^{2}(b)-\hat{\lambda}(b) \hat{\lambda}_{\text {MEAN }}(b)\right)$,

4. $\left.\sum_{b_{0}-1 b_{l}-1}^{B} \sum^{B} s\left(b_{0}\right) s\left(b_{l}\right) \frac{\partial^{2} l(\lambda)}{\partial \lambda\left(b_{0}\right) \partial \lambda\left(b_{l}\right)}\right|_{\dot{\lambda}}<0$ for all feasible directions of $s$.

The modified EM Algorithm in [20] can be ap- plied to find the MLE satisfying the KT conditions. Note that this is exactly the corresponding EM algorithm for the penalized likelihood in equation (7), not the One-Step-Late algorithm in [20]. The E-step needs to calculate

$$
\begin{aligned}
& Q\left(\lambda^{\text {new }} \mid \lambda^{d d}\right)-\alpha\left\|\lambda^{\text {new }}-\dot{\lambda}_{\text {MENN }}\right\|^{2} \\
& =-\sum_{d} \sum_{b} p(b, d) \lambda^{\text {new }}(b) \\
& \quad+\sum_{d} \sum_{b} \log \left\{p(b, d) \lambda^{\text {new }}(b)\right\} n^{\cdot}(d) \frac{p(b, d) \lambda^{\text {dd }}(b)}{\sum_{b^{-}-1}^{B} p\left(b^{\prime}, d\right) \lambda^{\text {dd }}(b)} \\
& \quad-\alpha\left\|\lambda^{\text {new }}-\dot{\lambda}_{\text {MFAN }}\right\|^{2},
\end{aligned}
$$

if we drop the term that does not involve $\lambda^{\text {new }}$. The Mstep needs to find $\lambda^{\text {mw }}$ that maximizes the above function. So, we look at the solution of

$$
\frac{\partial\left\{Q\left(\lambda^{\infty} \mid \lambda^{\alpha d}\right)-\alpha\left\|\lambda^{-\infty}-\dot{\lambda}_{\text {MEW }}\right\|^{2}\right\}}{\partial \lambda^{-\infty}(b)}=0 .
$$

Then, we have

$\frac{\hat{n}(b ; \cdot)}{\lambda^{m \omega}(b)}-\sum_{d=1}^{D} p(b, d)-2 \alpha\left[\lambda^{m \omega}(b)-\dot{\lambda}_{M E N}(b)\right]=0$,

where

$\hat{n}(b, ;)=\lambda^{\text {old }}(b) \sum_{d=1}^{D} \frac{p(b, d) n^{*}(d)}{\sum_{b^{\prime}=1}^{B} \lambda^{\text {old }}\left(b^{\prime}\right) p\left(b^{\prime}, d\right)}$.

That is,

where

$$
A\left[\lambda^{n+w}(b)\right]^{2}+B \lambda^{n+w}(b)-C=0,
$$

$$
\begin{aligned}
& A=2 \alpha, \\
& B=p(b ;)-2 \alpha \dot{\lambda}_{\text {MEN }}(b), p(b ;) \\
& C=n(b ;) .
\end{aligned}
$$

There are two possible roots of the above equation and the unique nonnegative root is

$$
\lambda^{n w w}(b)=\frac{-B+\sqrt{B^{2}+4 A C}}{2 A} .
$$

The resulting modified EM algorithm can be stated as follows.

Algorithm 3.1:

1. Choose initial values $\lambda^{\text {old }}(b)>0, b=1,2,3, \ldots, B$.

2. Compute a new estimate $\lambda^{\text {new }}(b)$ by (19) for $b=1$, $2,3, \ldots, B$.

3. If $\boldsymbol{\Phi}\left(\lambda^{\text {new }}\right)-\Phi\left(\lambda^{\text {old }}\right)$ is smaller than a tolerance, then stop. 
Otherwise, go to step 2 with $\lambda^{\text {old }}$ replaced by $\lambda^{\text {new }}$.

The computation cost and complexity for the proposed modified EM algorithm of CRMLE is of the same order as those of the MLE-EM. The convergence can be assured for any $\lambda_{\text {MEAN }}$ because we only need to shift the origin to $\lambda_{\text {MEN }}$ and use the Proposition in Green [20]. Thus, the monotonic convergence also holds for the modified EM algorithm of CRMLE. That is, the computation advantages of the EM algorithm are intrinsic to the modified EM algorithm in finding the CRMLE. The finite sample behaviors of CRMLE and the selection of penalty parameters are explored in the following section.

\section{IMPLEMENTATION AND DISCUSSIONS}

In contrast to the selection of a good iteration number such that the log-likelihood of MLE-EM is close to that of the true phantom as in Figure 2 for the MLE-EM, the more crucial factor for the CRMLE is selection of the penalty parameter. The finite sample behaviors of CRMLE depend heavily on the choice of penalty parameters. The log-likelihood of the mean estimate is usually smaller than those of the truc phantom and MLE as one can see in Figure 8. This is becausc the incomplete boundaries will oversmooth the image and pull down the log-likelihood to be below that of the true phantom. Since the log-likelihood of the true phantom lies somewhere in between those of MLE and mean estimate, it is reasonable to use the

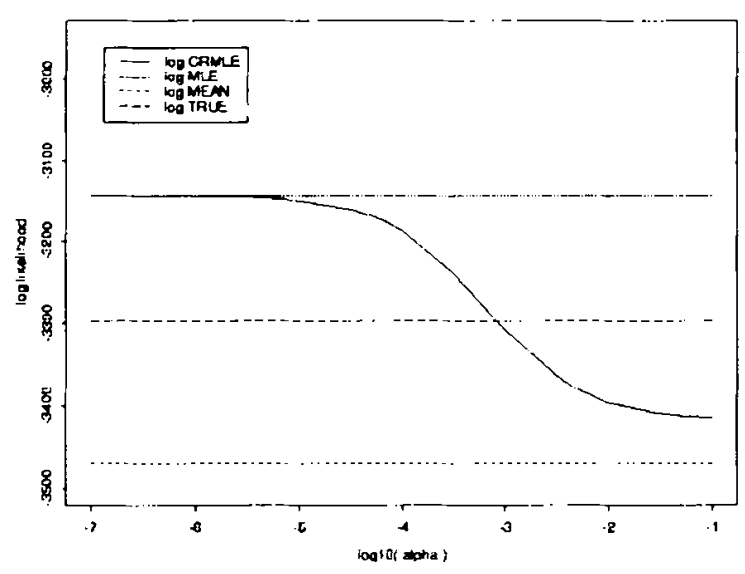

Fig. 8 The log-likelihoods of the CRMLE-EM, the converging MLE, the mean estimate and the true phantom with respect to penalty parameters are displayed. mean estimate as a reference point in the framework of MLE. As $\alpha \rightarrow 0$, the log-likelihood of CRMLE will approach that of the MLE. But if $\alpha \rightarrow \infty$, the $\log$-likelihood of the CRMLE will decrease to that of the mean estimate. Thus, with a proper choice of $\alpha$, the log-likelihood of CRMLE would be about the same as that of the true phantom. If the discrepancy measure is changed from the log-likelihood differences to the 2-norm differences with respect to the true phantom, the influences of penalty parameters in terms of 2-norm differences are shown in Figure 9. If the penalty parameter is chosen in the neighborhood of minimum point, 0.0005 , the CRMLE can indeed beat the mean estimate and MLE both numerically in Figure 9 and visually in Figure 7. The optimal values of $\alpha$ may be slightly different in Figure 8 and 9 owing to the difference of discrepancy measures.

In practice, the true phantom is unknown and the optimal value of $\alpha$ is impossible to obtain from the plots in Figure 8 or 9. One may consider a dynamic graph function, such as a scrolling box or slider, to choose a proper $\alpha$ with user's interactions. Another way is to reroute to an automatically data-driven choice of penalty parameters. Leave-one-out crossvalidation is one possible way. The idea is to remove one observation at a time. The remaining observations can be used to estimate the parameters. The fitted parameters can be applied to predict the value that is omitted. The sum of prediction square errors would depend on the penalty parameters. The minimum value of the sum of prediction square errors can provide a suitable choice for the penalty parameters. This method can be generalized to the transformed model of

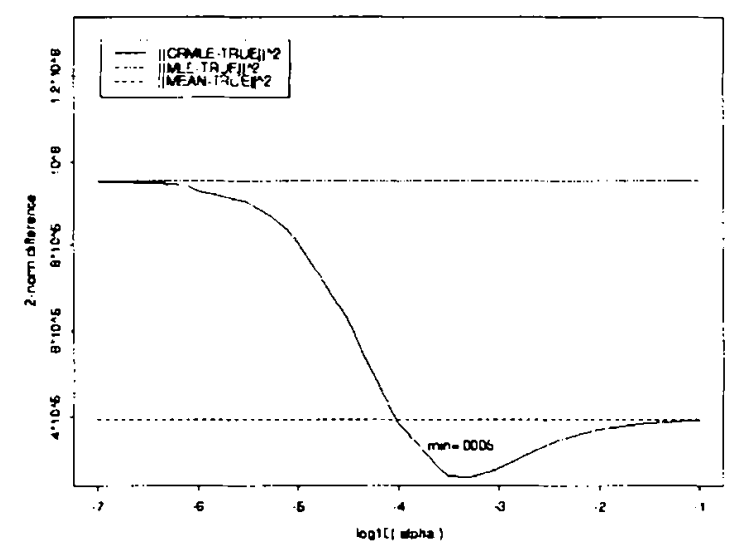

Fig. 9 The 2-norm differences of the CRMLE-EM, the converging MLE, the mean estimate and the true phantom with respect to penalty parameters are displayed. 
ridge regression such that the resulting choice of penalty parameters is rotational invariant. This is called as the generalized cross-validation (GCV) [23]. The GCV function of the ridge estimate for the PET reconstruction may be derived as

$$
V(\alpha)=\frac{\frac{1}{D} \sum_{i=1}^{D}\left(\frac{D \alpha}{d_{i}+D \alpha}\right)^{2} z_{i}^{2}}{\left\{\frac{1}{D}\left[\sum_{i=1}^{D} \frac{D \alpha}{d_{i}+D \alpha}+\left(D-D^{\prime}\right)\right]\right\}^{2}},
$$

where $M=[p(b, d)]^{\mathrm{T}}, d_{i}$ together with $u_{i}, i=1,2, \ldots, D$, are the eigenvalues and eigenvectors of $M M^{\mathrm{T}}, D$ is the rank of $M M^{\mathrm{T}}, z_{i}=u_{i}^{\mathrm{T}} y^{*}$, and $y^{*}(d)=n^{*}(d)-\sum_{b} p(b, d) \bar{\lambda}_{\text {MEAN }}(b)$. The GCV function against the penalty parameters is plotted in Figure 10. The minimum value, 0.0002 , is slightly different than the minimum values, 0.0005 , in Figure 8 and 9. The CRMLE with a proper penalty parameter selected in Figure 10 is shown in Figure 7. These two reconstructions do not differ too much in quality. If the boundary information is complete and correct, then the mean estimate will be a very good estimate. However, if the boundary information is incomplete or incorrect, then the mean estimate will oversmooth and mask the local fine structures. Via the CRMLE with a proper penalty parameter, we can keep the local fine structures and make use of the boundary information at the same time. The resulting reconstruction image in Figure 7 is very promising.

In calculating the GCV function, the eigenvalues and eigenvectors of $M M^{\mathrm{T}}$ are needed. The eigenvalue or singular value decomposition (EVD or SVD) for a matrix, symmetric or not, can be obtained by the imsl_f_eig_sym or imsl_f_lin_svd_gen functions in the IMSL C/MATH library for small- to medium-size matrices. Due to the limit of hardware, the above function does not work for large-size matrices. The power

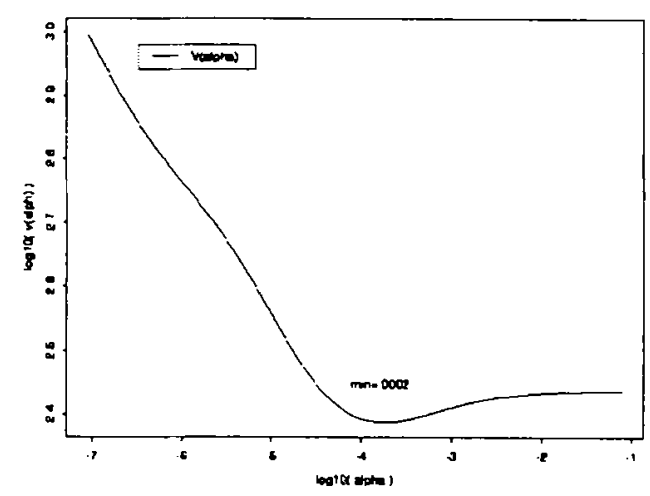

Fig. 10. The GCV curve with respect to penalty parameters is drawn method together with deflation [24] can be applied then to obtain the EVD or SVD. The eigenvalues obtained by the IMSL and power methods are not the same. To check the correctness of these two approaches, we can plot the error of $\left\|M M^{T} u_{i}-d_{i} u_{i}\right\|$, in 2-norm, for all eigenvalues $d_{i}$ and eigenvectors $u_{i}$. We find that the IMSL function does not get the right EVD at the beginning part and does a good job at the ending part. The power method is on the contrary. Therefore, if the leading part of EVD is needed, the power method is proper. But if the ending part of EVD is important, then the IMSL method is suitable. An improved numerical technique dominates the IMSL and power methods will be certainly useful in order to find the correct GCV. The EVD is quite computation demanding. But it is only needed to be done in one time since the matrix $M=[p(b, d)]^{\mathrm{T}}$ is fixed once the configuration of PET system is set up.

If the boundaries are not only incomplete but also incorrect due to misalignment, the mean estimate will be even far away from the true phantom. However, the CRMLE can still pull out the useful information with unsharp boundaries for those incorrectly specified boundaries. That is, the CRMLE can distinguish the correctly and incorrectly specified boundaries automatically. For those correctly specified boundary information, the CRMLE makes full use of the information because the mean estimate and MLE agree with each other. On the other hand, for the incorrect boundary information, the CRMLE recognizes the incorrectness and finds a good balance point between the mean estimate and MLE. If the boundaries are severely incomplete and incorrect, the mean estimate may even have larger 2-norm differences with respect to the true phantom than that of the MLE. The CRMLE can still outperform the MLE and the mean estimate. For instance, if the boundaries are so incomplete that there are only a half circle available in Figure 11, the mean estimate will lose the other half part of an ellipse.

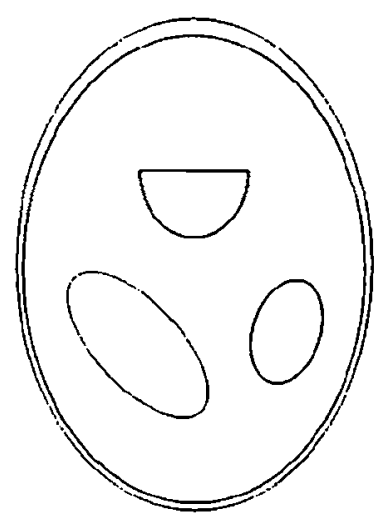

Fig. 11. The incomplete boundary information is shown. 


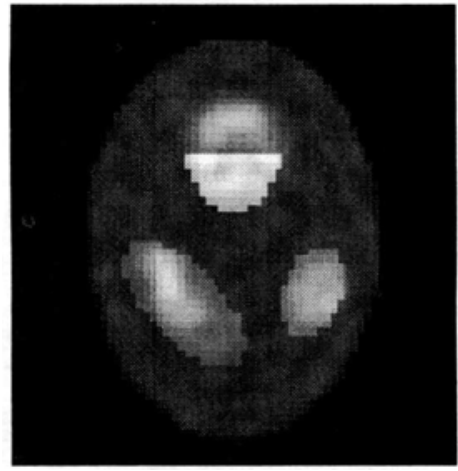

Fig. 12 The CRMLE-EM reconstruction is displayed with penalty parameter 0.0003 and 15 iterations.

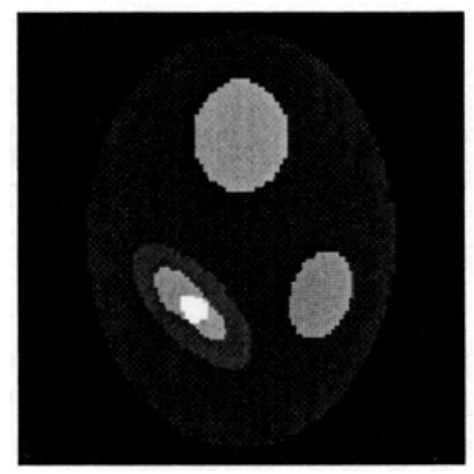

Fig. 13 Test phantom 2 is shown.

However, the CRMLE will make up the lost part as in Figure 12. The parameter, $\alpha=0.0003$, in Figure 12 is chosen because it minimizes both the GCV and 2norm difference curves. The distinguished capability in extracting the useful portion of the severely incomplete and incorrect boundary information has been observed in many other experiments for CRMLE, which are not shown in this paper for succinctness. To see the above phenomena in a higher resolution, the number of boxes is raised to $N_{b}{ }^{*} N_{b}=128 * 128$ and the number of detectors increases to $N_{d}=128$. We also illustrate this in a different test phantom as in Figure 13. An extreme case is that we may have incomplete boundaries that have holes as in Figure 14. The boundaries may be incorrectly located as well. The mean estimate is quite far away from the test phantom 2 in Figure 15. For the parameter, $\alpha=0.0005$, that minimize the 2-norm differences, the reconstruction of CRMLE is shown in Figure 15. The CRMLE can even fill up the holes when the penalty parameter is small enough, say $\alpha$ $=0.0002$. In any case, the CRMLE is quite robust to the misspecification and misalignment of boundaries. If the boundaries are complete and correct, then the mean estimate is a proper estimate. But if there is any doubt about the specification and alignment of boundaries, one shall use the CRMLE to reduce the

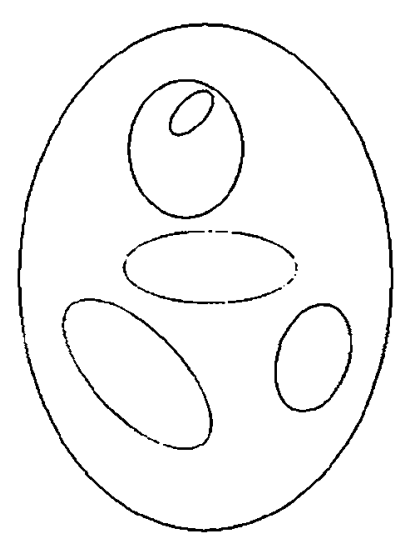

Fig. 14 The incomplete boundary information is displayed.

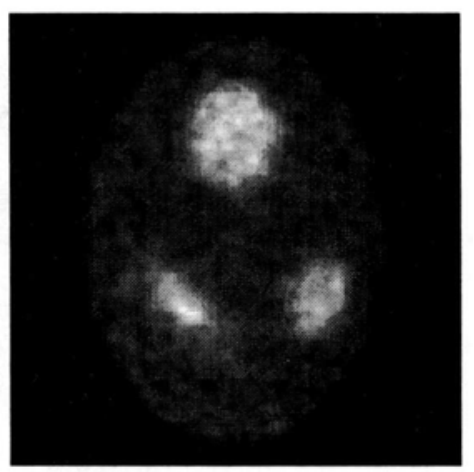

Fig. 15 The CRMLE-EM reconstruction is displayed with penalty parameter 0.0005 and 10 iterations.

effects of misspecification and misalignment.

\section{CONCLUSIONS AND FUTURE WORKS}

It has been shown in this study that the proposed CRMLE may take advantage of incomplete or incorrect boundary information effectively. By introducing the penalty parameters, one can do the dedicatory balance between the likelihood function and local smoothness within boundaries. By the modified EM algorithm derived, the computation speed and cost are of the same order as the MLE-EM. This makes the CRMLE-EM computationally feasible. Through either human intcractions or the generalized cross-validation method, we can choose a suitable penalty parameter to do the fine tune-ups. The Monte Carlo studies demonstrate the improvements of the CRMLE over the MLE. While the simulation results have confirmed that the CRMLE is a very promising approach, further studies are definitely required to make this scheme clinically 
applicable. Some important topics are how to register the boundary information from other imaging modalities on a PET, how to estimate the boundary if no other imaging modality is available, how to select penalty parameter more efficiently, etc. In addition, we would like to apply the proposed CRMLE algorithm to the real PET images as the next step.

\section{ACKNOWLEDGMENTS}

The authors would like to thank Professors I.-S. Chang, C. A. Hsiung, B. M. W. Tsui and W. H. Wong for their helpful discussions. This work was supported in part by the National Science Council of Taiwan, R. O. C.

\section{REFERENCES}

1. Luo WA: Incorporating Sactial-Invariance Feature in Filter-Backprojection Algorithms. Master Thesis, National Taiwan University, 1996.

2. Shepp LA and Vardi Y: Maximum Likelihood Reconstruction for Emission Tomography. IEEE Trans Med Imaging 1982; 1: 113-122.

3. Lange $\mathrm{K}$ and Carson R: EM Reconstruction Algorithms for Emission and Transmission Tomography. J Comput Assist Tomog 1984; 8: 306-316.

4. Vardi Y: Network Tomography : Estimating Source-Destination Traffic Intensities from Link Data. Journal of the American Statical Association 1996; 91(433): 365-377.

5.Politte DG and Snyder DL: Corrections for Accidental Coincidences and Attenuation in MaximumLikelihood Image Reconstruction for PositronEmission Tomography. IEEE Transactions on Medical Imaging 1991; 10(1): 82-89.

6. Fessler JA, Clinthorne NH and Rogers WL: On Complete-Data Spaces for PET Reconstruction Algorithms. IEEE Transactions on Nuclear Science 1993; 40(4): 1055-1061.

7. Snyder DL and Miller MI: Random Point Processes in Time and Space. Springer, $2^{\text {nd }}$ Ed., 1991.

8. Joyce LS and Root WL: Precision Bounds in Supperresolution Processing. J. Opt. Soc. Am. A 1983; 1: 149-168.

9. Lu HHS and Wells MT: Minimax Theory for Latent Function Estimation. Technical Report, National Chiao Tung University, 1994.

10. Lu HHS and Wells MT: Adaptive Constrained Method of Regularization Estimators for Latent Density Estimation. Technical Report, National Chiao Tung University, 1994.
11.Snyder DL, Miller MI, Thomas LJJ, and Politte DG: Noise and Edge Artifacts in Maximum-Likelihood Reconstructions for Emission Tomography. IEEE Transactions on Medical Imaging 1987; 6: 228-238.

12. Veklerov E and Llacer J: Stopping Rule for The MLE Algorithm Based on StatisticalHypothesis Testing. IEEE Trans. on Medical Imaging 1987; 6(4): 313-319.

13. Snyder DL and Miller MI: The Use of Sieves to Stabilize Images Produced with The EM Algorithm for Emission Tomography. IEEE Transactions on Nuclear Science 1985; NS-32(5): 3864-3872.

14. Hebert T and Leahy R: A Generalized EM Algorithm for 3D Bayesian Reconstruction from Poisson Data Using Gibbs Priors. IEEE Trans. on Medical Imaging 1989; 8(2): 194-202.

15. Green PJ: Bayesian Reconstructions from Emission Tomography Data Using A Modified EM Algorithm. IEEE Trans. Med. Imaging 1990; 9: 84-93.

16. Herman GT, Pierro ARD, and Gai N: On Methods for Maximum A Posteriori Image Reconstruction with A Normal Prior. Journal of Visual Communication and Image Representation 1992; 3(4): 316324.

17. Ouyang X, Wong WH, Johnson VE, Hu X, and Chen CT: Incorporation of Correlated Structural Images in PET Image Reconstruction. IEEE Transactions on Medical Imaging 1994; 13(4): 627-640.

18. Vardi Y, Shepp LA, and Kaufman L: A Statistical Model for Positron Emission Tomography. Joumal of the American Statical Association 1985; 80: 820.

19. Silverman BW, Jones MC, Wilson JD, and Nychka DW: A Smoothed EM Approach to Indirect Estimation Problems, with Particular Reference to Stereology and Emission Tomography. J. R. Statist. Soc. 1990; B-52(2): 271-324.

20. Green PJ: On Use of The EM Algorithm for Penalized Likelihood Estimation. J. Roy. Statist. Soc. 1990; B-52(3): 453-467.

21.Johnstone IM and Silverman BW: Speed of Estimation in Positron Emission Tomography and Related Inverse Problems. Ann. Statist. 1990; 18(1): 251-280.

22. Geman S and Geman D: Stochastic Relaxation, Gibbs Distribution, and The Bayesian Restoration of Images. IEEE Trans. Pattern Anal. Machine Intell. $1984 ; 6:$ 721-740.

23. Golub GH, Heath M, and Wahba G: Generalized Cross-Validation As A Method for Choosing A Good Ridge Parameter. Tchnometrics 1979; 21(2): 215-223.

24. Parlett BN: The Symmetric Eigenvalue Problem. Prentice-Hall, 1980. 